\title{
Towards Progress Indicators for Measuring Student Programming Effort During Solution Development
}

\author{
Stephen Edwards \\ Department of Computer Science \\ Virginia Tech \\ Blacksburg, VA, USA 24061 \\ edwards@cs.vt.edu
}

\author{
Zhiyi Li \\ Department of Computer Science \\ Virginia Tech \\ Blacksburg, VA, USA 24061 \\ zli04@cs.vt.edu
}

\begin{abstract}
When learning to program, assignment feedback can easily reinforce a fixed mindset - where one believes intelligence is a fixed ability you either have or you don't. However, this can have negative consequences for learning. The alternative is a growth mindset, where one believes intelligence is malleable and can be improved through practice, effort, and hard work. We develop a set of fifteen progress indicators that can be used to assess student programming effort independently of the correctness of their code. The goal is to provide the measurement support needed for innovative feedback that gives a more welcoming experience for students, recognizing the effort they put in and the accomplishments they make as they work on solutions, rather than simply looking at whether code "works". Our set of progress indicators includes seven associated with writing solution code for a problem that are suitable for use on all programming assignments, and eight associated with self-checking programs using software tests, which are appropriate for assignments where students are required to test their own work. In this initial work, we applied these indicators to a collection of programming assignments from 257 students to determine the suitability of the measures and validate their results. The resulting set of progress indicators is the first step toward developing feedback strategies that recognize and reward effort and hard work, with the goal of fostering the development of a growth mindset among students.
\end{abstract}

\section{CCS Concepts}

-Social and professional topics $\rightarrow$ Student assessment; -Applied computing $\rightarrow$ Computer-assisted instruction; •Software and its engineering $\rightarrow$ Software development techniques;

\section{Keywords}

growth mindset; fixed mindset; metrics; indicators; progress; effort; persistence; feedback; automated grading

Permission to make digital or hard copies of all or part of this work for personal or classroom use is granted without fee provided that copies are not made or distributed for profit or commercial advantage and that copies bear this notice and the full citation on the first page. Copyrights for components of this work owned by others than the author(s) must be honored. Abstracting with credit is permitted. To copy otherwise, or republish, to post on servers or to redistribute to lists, requires prior specific permission and/or a fee. Request permissions from permissions@acm.org.

Koli Calling 2016, November 24 - 27, 2016, Koli, Finland

(C) 2016 Copyright held by the owner/author(s). Publication rights licensed to ACM. ISBN 978-1-4503-4770-9/16/11 . \$15.00

DOI: http://dx.doi.org/10.1145/2999541.2999561

\section{INTRODUCTION}

Like many universities, our department struggles with attrition issues in our early courses. Despite a number of interventions to increase motivation, we still see too many capable and interested students start out excited about computer science, only to become discouraged and quit. One hypothesis is that such students just "think they don't have what it takes" to succeed in computing. When a student believes his or her intelligence is a fixed asset, they have a fixed mindset. However, students with such beliefs are more likely to avoid academic challenges, perform more poorly, and give up sooner than students with a growth mindset - students who believe that they can increase their abilities through hard work and effort [11]. Individuals with a fixed mindset tend to focus on performance goals (points), be-cause they equate academic achievement with an indication of their intelligence or ability. This is in contrast to growth mindset individuals, who are more likely to adopt learning goals (concept mastery) that challenge them to increase their own abilities.

Unfortunately, developing a growth mindset for students is hard, even harder for students when their teachers hold a fixed mindset [24]. Lewis [22] found that $77 \%$ or more of CS faculty disagree with the statement that "Nearly everyone is capable of succeeding in the computer science curriculum if they work at it." Thus, it is unsurprising that classroom assessment approaches in computing focus on purely objective, points-driven scoring, with feedback that is framed in the negative about what features do not work, what points were lost because of mistakes, or what software tests do not pass. The problem is further exacerbated by automated grading tools, which are typically coldly objective in assessing the correctness of student programs, without any recognition of the effort or hard work that went into constructing them. The feedback students receive tends to reinforce performance-oriented goals, and fails to meaningfully support learning goals. Students may have initial experiences with automatic grading in computing courses that they perceive as cold, impersonal, depressing, or discouraging. Facing a zero score accompanied only by complaints about what doesn't work in a program is a sobering experience that can "turn off" students. While the evaluation may reflect the truth in regards to the end product, it is too easy for students to interpret such an assessment as a value judgment on their own abilities, and on the work and effort they have invested to get to that point in their solution process. In short, it is too easy for students to interpret such feedback as a discouraging slap in the face that says "you don't have it", and that encourages thoughts of giving up. 
This paper presents initial work necessary for new approaches to automated feedback that provides students with a more positive, encouraging experience on assignmentsone that clearly recognizes hard work and effort and avoids the traps of purely objective, correctness-based feedback. We believe it will eventually be possible to provide feedback that encourages and strengthens beliefs in a growth mindset, and that communicates to students that persistence and hard work are valued traits. However, before reaching point, we first have to address the question of how we can appropriately measure "effort" in order to meaningfully drive feedback generation. Unfortunately, measuring effort or hard work in a student's solution process is difficult.

Here, we describe a suite of fifteen indicators that can be automated and that reflect student effort on an assignment. While no single indicator may reliably measure effort, we use triangulation of multiple indicators to provide evidence of level of effort, and to prevent simple "gaming of the system" by students. We then validate these indicators against historical data collected from past courses and describe the results. Based on this suitability evaluation, we find that the set of fifteen indicators has utility for identifying student effort independently of scores earned on an assignment. Further, the indicators provide the measurement support necessary for future efforts to generate positive feedback for students designed to encourage a growth mindset.

\section{BACKGROUND AND RELATED WORK}

\subsection{Fixed Versus Growth Mindsets}

The concepts of entity theory (fixed mindset), incremental theory (growth mindset), and related self-theory (selfconcept) originated from the psychology research community. Dweck [11] describes self-theories of intelligence-one's personal beliefs about one's own intelligence and how it develops. A fixed mindset is a self-theory that one's intelligence is a fixed, pre-determined feature of the individual. In contrast, a growth mindset is a self-theory that one's intelligence can grow and improve through practice and learning. Corresponding motivational frameworks for both mindsets indicate they affect an individual's behavior and thinking, leading to differences in motivation, reactions to failure, and academic achievement [4].

Individuals who believe in a fixed mindset - that their intelligence is an innate and unchanging ability - tend to avoid challenges, ignore or reject criticism, and focus on performance outcomes. Performance, as indicated by grades, may be interpreted as external validation that their believedinnate intelligence is sufficient, while low grades or criticism may be seen as indicating insufficiency. This leads fixed mindset students to react more negatively to criticism or academic struggle, and to consider giving up or changing track as challenges increase. Individuals who believe in a growth mindset - that their intelligence develops with practice and hard work - have a different view on learning. They tend to view challenges as ways to develop and strengthen their skills, and are more likely to focus on mastering learning rather than just grades. Growth mindset individuals are more likely to interpret criticism as an indicator they should work harder. There are clear differences in motivation, academic outcomes, and willingness to seek help between these two perspectives, with individuals possessing a growth mindset tending toward more positive learning $[4,10,12,17]$.
Fortunately, interventions have shown that mindsets can be changed, improving academic performance as a result [4]. For members of underrepresented groups, changing to a growth mindset also appears to reduce susceptibility to stereotype threat [2]. This is particularly important in engineering, where women are more likely to hold a fixed mindset [19], resulting in a greater tendency to drop courses in response to academic difficulties. Self-theories of intelligence have already been suggested as a possible cause of the underrepresentation of women in computing [15].

\subsection{Mindset Interventions in Computer Sci- ence Education}

Within computing education, researchers have investigated several interventions aimed at changing mindsets held by students [18, 23, 24, 27] with varying degrees of success. Hanks et al. [18] present a study analyzing advice given by students on how to succeed, where students were prompted to give advice that would encourage a growth mindset. In their study, the advisory comments produced by students were categorized into three groups: general study advice, programming-specific advice, and attitudinal advice. General advice $(63 \%)$ was given more frequently than programming-specific $(23 \%)$ or attitudinal advice (34\%), even though students are prompted to encourage future students to develop a growth mindset towards programming. Their study does not show conclusive statistical results with respect to shifting mindsets. However, students' advice in their study help educators to understand how students think about what is necessary to succeed in programming. Lewis et al. [23] present a study of interviews with students in the introductory programming courses in two universities. The interviews with students are about their self-assessment to decide to major in computer science. One of the factors studied was a student's CS-related ability. Students rate their CSrelated ability based on previous programming experience, speed to finish tasks, and grades of computer science courses. Extended from Dweck's [11] self-theory, their study shows students' interpretation of CS-related ability is influenced by belief about whether this ability is fixed or malleable.

In terms of interventions, Simon et al. [27] apply "Saying is believing" exercises in CS1/CS2 to see whether they can shift students' mindset toward a growth perspective. "Saying is believing" [20] is an intervention to ask students to advocate a growth mindset by relating to their own experiences. However, no statistically significant differences were found between the experimental and control group in their study. They analyzed possible explanations including strength of interventions and threats to validity. Murphy et al. [24] investigate the implicit influence of self-theories research on computer science education. They found that fixed mindset beliefs are a danger to success for computer science students. In computer science education, students often face challenges, and whether they have a fixed mindset or malleable mindset can seriously affect learning to program. Further, these effects may contribute to decisions about choosing CS as a major, particularly for women and other underrepresented groups. Their study on self-theories research related to computer science education suggests CS teachers can move students toward malleability when students handle different tasks: known tasks, attainable tasks, and unattainable tasks by providing a malleable view of tasks. For example, students can be helped in their tasks 
with scaffolding, or through targeted help and guidance from a teacher or peer.

However, the shift of students' fixed mindset to growth mindset is not automatic. Dweck recently gave a warning for overextending her work on growth mindsets in her blog [9]. She claims that rewarding or praising students only for their effort, not for learning, is a misapplication of growth mindset research. In her blog, Dweck first admits that human beings typically hold a mixture of fixed and growth mindset beliefs, but can move closer to a growth mindset with thoughts and practices. Educators should adopt a deeper, true growth mindset perspective in their classroom practices by concentrating on the student learning process.

Regarding encouraging internal belief changes, Deci et al. [8] presented a review of experiments about the effects of extrinsic rewards on intrinsic motivation of children and college students. Extrinsic rewards are defined into two categories: tangible rewards such as money, and verbal rewards such as praise. They find whether the rewards will enhance or undermine intrinsic motivation depends on many elements such as interpersonal contexts like homes, classrooms, or workgroups; quality of the tasks such as interesting or boring; rewards categories such as expected tangible, unexpected tangible, task-noncontingent, or verbal rewards; measures of effects; and age differences. Their review shows positive feedback enhance intrinsic motivation in some situations. They find evidence that the strategies to use extrinsic rewards have a serious risk of diminishing instead of promoting people's intrinsic motivation if used incorrectly.

Abraham E. Flanigan et al. [16] investigated how students' implicit theories of intelligence change in CS1 across the whole semester. They applied the Implicit Theories of Intelligence Scale [11], which uses eight Likert-scale items to measure students' implicit theories of intelligence. Their study found that more students hold a growth mindset compared to those with a fixed mindset across the semester. However, they also documented a significant decrease in growth mindset, and a corresponding significant increase in fixed mindset. As a result, Flanagan et al. recommend that CS educators concentrate on sustaining student beliefs in a growth mindset. Barker et al. [3] present a study of the learning environment in computer science classrooms. In this learning environment, communication patterns between teacher and students and among students are important in the learning process. They find computing classrooms have environments that are more impersonal, with more guarded, competitive behavior from students, due to the characteristics typical of CS majors. These communication patterns can lead to a defensive climate in the classroom. This defensive climate may impede the development of collaborative and supportive learning behaviors.

These approaches are beneficial in many ways. At the same time, however, current work has not addressed one of the primary classroom mechanisms for individualized criticism: the feedback students receive on assignments, which is an important component of a collaborative and supportive learning environment. Because students with different mindsets can react to and interpret the same forms of criticism in very different ways, it is important to consider how assignment feedback affects student thinking and behaviors. Self-theories of intelligence provide a unique theoretical lens for considering the implication of feedback.

One of the most difficult challenges in fostering mindset change is assessing student effort, or "hard work" in students' learning process. To provide growth mindset feedback, it is necessary to measure how well students are engaging in the activities that promote intellectual growth. The approach taken in this research is heavily inspired by the work of O'Rourke et al. [25, 26] on creating game incentives that promote a growth mindset. They describe Refraction, a video game designed to teach fractions to children, and how they developed a system of "brain points" to measure student effort and provide feedback messages intended to promote a growth mindset. Their system of measuring student work is particularly relevant, since it is difficult to construct a single measure that captures the ideal reliability. Instead, O'Rourke's team used an approach where they created a collection of four simple, but less reliable, indicators. No single indicator was a perfect measure of effort, but each indicator was expected to be present under some circumstances where students were investing effort in trying to solve problems. To indicate students had applied effort, the game required positive results on at least two of the indicators - a simple form of triangulation. A similar concept has been frequently used in other measurement contexts, particularly with software quality metrics, where corroborative evidence from multiple weak metrics can be used to obtain more consistent and meaningful results than any one metric alone.

\subsection{Current Practice in Automated Feedback}

While there is a long history in automatically assessing programming work in the classroom - a recent survey is provided by Ihantola et al. [21] — there has been much less work done on the design of feedback provided by such tools. Many grading tools have feedback strategies that grow organically based on technical solutions or instructor needs, without any theoretical underpinnings. The primary motivation of feedback is often to provide scoring results, usually with the goal of providing these results to students so that they can rework and resubmit to achieve better outcomes. Unfortunately, the focus is very grade-oriented, which only reinforces the idea among students that grade performance is the primary concern, and therefore the primary goal. This focus tacitly reinforces the fixed mindset perspective.

As an example, Figure 1 shows part of feedback produced by Web-CAT $[1,13,14]$, a widely used open-source automated grading system. The score summary visible in Figure 1 summarizes the points earned from automatic scoring, and also provides a placeholder for the portion of the grade assigned through manual inspection. In interviews, students report that the green/red "progress bars" that graphically show the points earned are the most important pieces of information on the page. Since Web-CAT permits students run their own test cases for their code, if students test their own code, similar green/red bars are shown alongside each file in the student's submission, indicating how thoroughly it has been tested.

Unfortunately, it is extremely common for students to receive a score of zero on their first submission (or first several submissions). This actively discourages students from using automated assessment until they are close to finish an assessment. It also has a negative impact on student morale and does nothing to promote a sense of accomplishment as students are approaching completion of an assignment.

As students progress through an assignment, assessment systems such as Web-CAT usually provide feedback about 


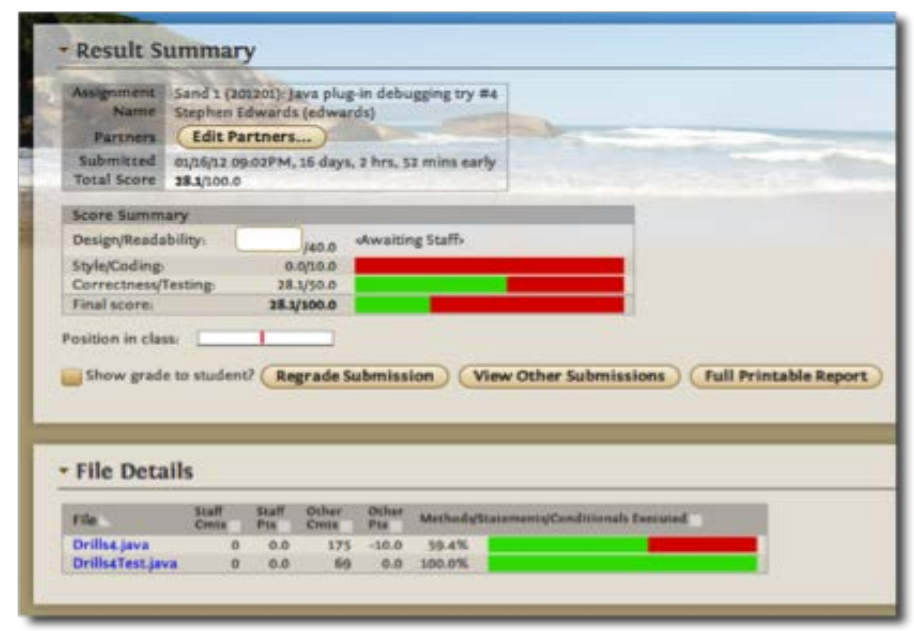

Figure 1: A score summary in Web-CAT.

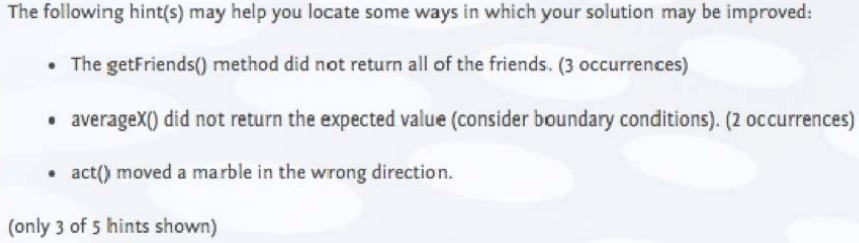

Figure 2: An example of behavioral feedback.

software faults. Systems that assess student work by comparing output often show a test input sequence (or fragment) along with the actual output produced by the student's program, and expected (correct) output. Systems that use other forms of test cases may show raw test cases results, or simply show the name of test cases. Web-CAT typically uses software test cases to assess behavioral correctness of student work, but allows instructors to control the messages generated when (hidden, instructor-written) tests fail, and limit the results so that students are discouraged from using the assessment system as the sole means of testing their own work. Figure 2 shows an example of what students might receive as feedback on the behavioral errors in their work. Messages are instructor-written and embedded in the software tests, allowing instructors the freedom to choose how detailed (or general) each message is. However, writing feedback messages is time-consuming, and writing messages that correctly report problems is difficult. It is easy for instructors to forget that when a test for feature $\mathrm{X}$ fails, that does not necessarily imply there is a bug in the implementation of feature $\mathrm{X}$-instead, the bug could be somewhere else entirely, but was simply observed when trying to make use of feature X. While software tests can be extremely useful for exposing the presence of bugs, identifying the location of the bug usually involves more than just knowing which test case failed. Messages that attempt to diagnose problems can often end up misdirecting students, since one test may actually fail due to many alternative bugs besides the primary failure targeted by the writer of the test.

By focusing on points earned and on failures or errors observed, feedback received by students is largely negative.
Some work has been done on providing more positive feedback [5, 6, 7], but has not seen widespread use. While the feedback characterized here - and that of most automated assessment systems - is objective and truthful, it presents a cold, impassionate face to students that delivers negative, performance-oriented feedback. It has the potential to reinforce fixed mindset views. At the same time, conventional feedback misses the opportunity to positively reinforce desired development strategies.

\section{PROGRESS INDICATORS}

The first step in generating growth mindset-oriented feedback is measuring a student's hard work and productive effort in the learning process. Inspired by the work of $\mathrm{O}^{\prime}$ Rourke et al. [25, 26], we define a set of progress indicators instead of a single measure. While each individual indicator may be noisy and fragile, their results will be combined using a simple decision rule to produce a more robust composite measure. Each individual indicator is intended to capture a different aspect of productive work on a programming assignment. Some students might "hit" one particular subset of indicators at one point, or a completely different set later as they work on their assignment. The indicators triggered will vary from student to student, as well as over time.

\subsection{General Purpose Indicators}

The first seven indicators all apply to various aspects of constructing a software solution to a problem.

1. Adding New Solution Method(s): the student's solution contains more methods than before.

2. Removing Static Analysis Errors: problems in the layout, formatting, commenting, or style of the code have been resolved.

3. Reducing Cyclomatic Complexity: the student's solution contains fewer branches and/or methods than before.

4. Reducing Average Method Size: the average number of statements per method has been reduced.

5. Increasing Comments Density: the proportion of comment lines, relative to the number of source code lines, has increased.

6. Increasing Solution Classes: the student's solution contains more classes than before.

7. Increasing Correctness: the number of instructorprovided reference tests passed by the student's solution is more than before (the solution's behavioral correctness has increased).

Each of these indicators is phrased in terms of simple properties of the student's solution. Indicators 1-6 rely on counting basic features visible in the solution's source code and are easily automated. Indicator 7 is the only one that requires dynamic analysis, and it is based on information that is typically collected by automated grading systems.

Furthermore, each indicator is relative, based on comparing the current state of the solution to an earlier state. The idea is to look at a student's work over time in order to 
gauge whether progress is being made. If an existing automated grading solution is being used, we can simply leverage it to collect measures. Each time a student submits their work for checking, they are offering a snapshot of its current state. By comparing changes in measures between submissions, progress can be assessed.

For this study, the measures were implemented using WebCAT. Web-CAT's plug-in for automatically grading Java programs already collects a significant amount of static analysis data and dynamic analysis data from student code. The static program metrics collected by the plug-in were already sufficient to directly implement the seven measures described above without any additional tool programming.

\subsection{Software Testing Indicators}

The remaining eight indicators all apply to various aspects of writing software tests to accompany a solution, as some students are required to do on some assignments. Instead of relating to progress on the solution, these indicators focus on progress in writing better software tests.

8. Adding New Test Method(s): the student's software test suite contains more tests than before, while the number of solution methods remains the same.

9. Adding to Existing Tests: the number of statements in the student's test suite is greater than before, while the solution remains approximately the same size.

10. Increasing Number of Tests per Method: the number of test methods increases, relative to the number of methods in the solution.

11. Increasing Statement Coverage: the student's own software tests execute a larger number of statements in the solution than before.

12. Increasing Method Coverage: the student's own software tests execute a larger number of methods in the solution than before.

13. Increasing Conditional Coverage: the student's own software tests execute a larger number of conditional branches in the solution than before.

14. Increasing Assertion Density: the student's own software test execute a larger number of assertions per methods in test than before.

15. Increasing Test Classes: the number of test classes increases in the solution than before.

As with the first group of indicators, these eight consist of some that are based on static analysis of the source, while others are based on dynamic analysis of the running solution. Here, indicators 8-10 rely on counting basic features of the source code of software tests. Indicators $11-15$ rely on code instrumentation data collected as the tests execute. Again, all of these indicators are already directly supported by data collected by Web-CAT's plug-in for Java assignments.

\subsection{Applying Indicators Over Time}

While all of the indicators described here aim to capture very basic notions of progress that lead toward an improved solution, these simple indicators also share another property: they are imprecise and noisy. Virtually any kind of change to a student's work, whether it is productive or purely random, is likely to lead to changes in at least one of the indicators, possibly more. The indicators are imprecise because, even though they capture ideas that represent good behaviors, that can easily signal poor behaviors as well. For example, consider increasing comment density. When a student adds useful documentation comments to a method, the comment density will increase, representing a positive effort on the student's part. However, if the student takes a working method and deletes the statements from its body, that will likely degrade the solution. Still, by removing statements and leaving comments the same, the net density of comments has still increased.

Therefore, it is important to attempt to decrease the "noise" inherent in these indicators. For that, we will adopt a technique from statistics when dealing with noisy, time-series data by applying a finite impulse response filter on the measures. One of the most common such filters for time-series data is the rolling average (or moving average). The concept behind a rolling average is to average together the values of a signal or measure over an interval of time, which results in a smoothing effect that reduces noise. It produces a mean (or average) that is time-sensitive.

Instead of the mean of the previous $N$ values, we can consider the maximum of the previous $N$ values. For any indicator that is defined in terms of an increase of some property, we can examine the previous $N$ submissions and determine whether the current submission is an increase over the maximum from the previous $N$ submissions. When fewer than $N$ previous submissions exist, all previous submissions are used. By examining historic data from student submissions, $N=4$ provides an appropriate amount of smoothing. In other words, a particular feature (such as comment density) can be judged as increasing if it is greater than the value computed for the previous four submissions.

One advantage of this approach to smoothing data is that it also makes it hard for students to "game" the indicators. While there is the potential for students (if the indicators are widely known) to intentionally make changes to any one measure through simple manipulation of their source code, this becomes more cumbersome when individual changes are judged relative to a rolling maximum, rather than just relative to the previous submission.

\section{EVALUATING SUITABILITY}

While the proposed indicators are straightforward to define, it is important to evaluate how they operate on real student-written programs and whether they are effective at identifying effort. To that end, we conducted a feasibility study on student programs collected from two semesters of the CS2 course at Virginia Tech. Four individual programming assignments were offered each semester, involving a total of 257 students who made 20,363 program submissions, completing 983 separate program solutions. On average, a student submitted 20.8 attempts for each programming assignment. This course required students to write software tests for their work, so the full suite of fifteen indicators was 


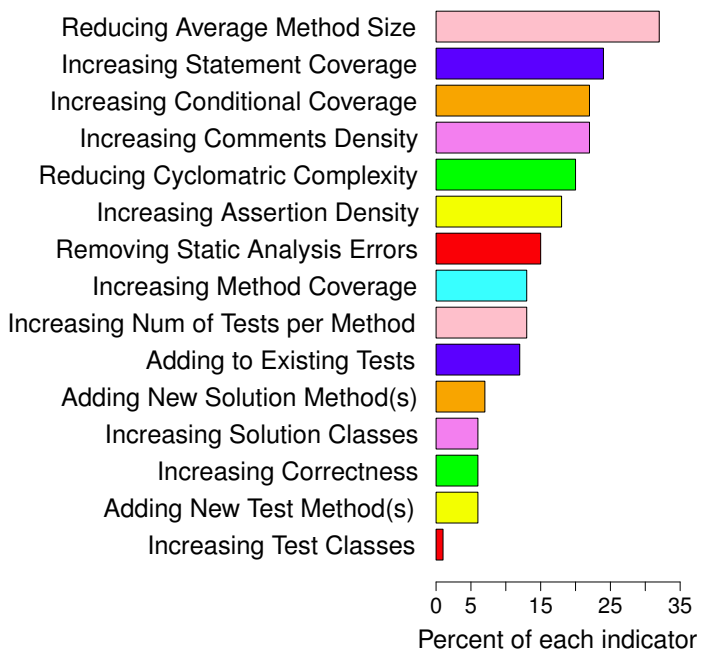

Figure 3: Proportion of submissions triggering each indicator.

applied to every submission in the dataset.

Figure 3 shows the frequency for each of the indicators, in terms on the total number of submissions satisfying the indicator. Individual indicators triggered on one third or fewer of the submissions overall, with the most common indicator being Reducing Average Method Size.

While the majority of submissions did not trigger a given indicator, we carefully examined the values that led to positive triggers on each indicator. Somewhat surprisingly, the indicators all exhibit a similar distribution shape, in terms of the the amount of "improvement" in one submission over the previous four. We consider Adding New Solution Methods as a representative example. Only $6.6 \%$ of submissions show an increase in the number of methods in their solution, compared to the previous four submissions. However, if we examine just those that have more methods, the number of new methods added is distributed as shown in Figure 4. Most submissions that add more methods add just a few, following roughly an exponential decay-a common pattern recurring across the indicators. Other metrics show the same basic distribution, although with varying scales depending on the nature of the indicator.

\subsection{A Composite Measure}

Even after smoothing, the indicators are individually imprecise. A variety of potential behaviors by students may cause an indicator to trigger, including both beneficial "hard work" by the student, and other unrelated behaviors, some of which might even be undesirable. In other words, a single indicator alone is likely to have a high error rate, potentially including both type I (false positive) and type II (false negative) errors. As an example, again consider Adding New Test Methods. If a student is productively working on a solution in a way that involves adding a new method that was not present before, then this indicator would normally trigger. However, it will also trigger when a student adds a poorly designed method that is not even used elsewhere in the solution (type I). Worse, if the student then refactors that method in a positive way so that it contributes mean-

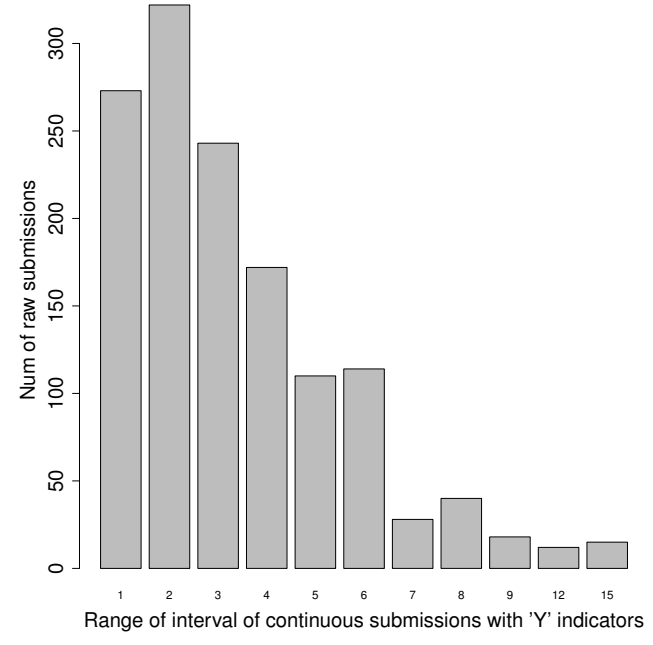

Figure 4: A representative example of one indicator's distribution, for Adding New Solution Methods.

ingfully to their design, the indicator will not trigger since no "new" method was added (type II). Looking at a single indicator in isolation can be quite inaccurate.

Accuracy may be improved via triangulation - that is, looking for when indicators corroborate each other. For example, while it is easy to find ways that any single indicator may produce incorrect results, we can also consider the hypothetical case where every indicator triggers. It is virtually impossible to construct a pathological scenario in which this happens where the student has not invested a significant amount of effort that also happens to move their solution forward. Indeed, because some indicators contradict each other - for example, adding a new method also increases cyclomatic complexity-looking for all indicators to simultaneously trigger would most likely eliminate type I errors at the expense of maximizing type II errors. Instead, a compromise is necessary.

Here we define a simple composite measure using the same approach as O'Rourke et al. [25, 26]: if at least $K$ indicators are present on a single student submission, that submission is judged to include productive work. Which value of $K$ is appropriate? Figure 5 shows the proportion of submissions with at least $K$ indicators, considering just indicators $1-7$ that are appropriate for any assignment. The values approximate a geometric distribution. Choosing a lower $K$ would consider more submissions as "making progress", while increasing false positives. On the contrary, a larger $K$ produces a different tradeoff, with fewer submissions judged as "making progress", at the cost of more false negatives.

Figure 6 shows the same chart when considering the entire suite of 15 indicators, which also approximates a geometric distribution. It is worth noting that no submissions triggered all indicators, with just one submission triggering 14 and six submissions triggering 13. Based on these figures, a $K$ between $5-8$ would produce positive progress judgements on an average of $5-15 \%$ of submissions when all fifteen indicators are used. Similarly, a $K$ between $3-4$ would produce similar behavior when just the first seven indicators are used. 


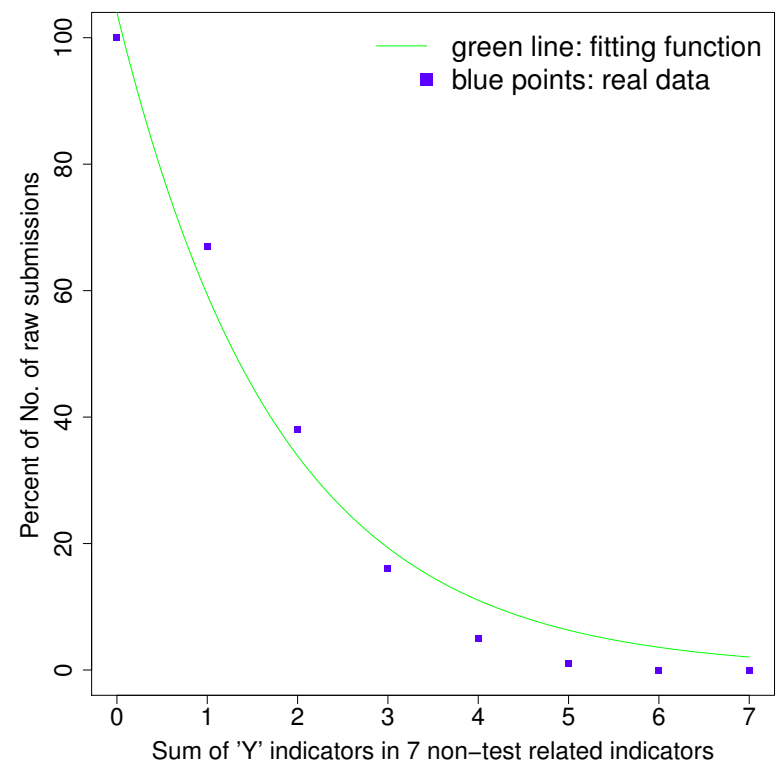

Figure 5: Percent of submissions with at least $K$ indicators, considering indicators 1-7.

One serious concern, however, is that indicators should measure productive effort, not simply number of submission attempts. If the composite measure is linearly related to the number of submissions, then students who simply make more submissions appear to "work harder", while classroom experience suggests the opposite. In practice, it is common to see students who are stuck "thrash" - that is, when stuck, a student may make a series of small changes without really understanding what the problem is, or whether the change resolves the problem, resulting in a larger number of submissions that are mostly nonproductive. There is a risk that, regardless of the measure chosen, a student who simply makes a larger number of submissions might appear to be making more progress, simply because more submissions means more opportunities to trigger the indicators.

To explore this issue, we examined the relationship between possible values of $K$ and the number of submissions made by students. Figure 7 uses a scatter plot to show the relationship for $K=6$ indicators, when all 15 indicators were applied. In Figure 7, each point represents an individual student's submission information: the horizontal axis represents the number of submissions with at least $K=6$ indicators triggered, while the vertical axis shows the total number of submissions the individual student made. Across all values of $K$, we found that lower $K$ values were associated with higher Spearman's rho correlation coefficients ( $\rho=0.97$ for $K=1$ ), while high values of $K$ were uncorrelated $(\rho=-0.1$ for $k=12)$. We eliminated from consideration $K$ values that had strong correlations $(\rho>=0.6)$ with the total number of submissions, which included all values between $K=1(\rho=0.97)$ through $K=5(\rho=0.69)$. The value of $K=6$ is the smallest to have only a moderate correlation with the number of submissions made $(\rho=0.57)$.

As shown in Figure 7, the majority of the students in this exploratory analysis have from $4-37$ submissions ( $\mu \pm$

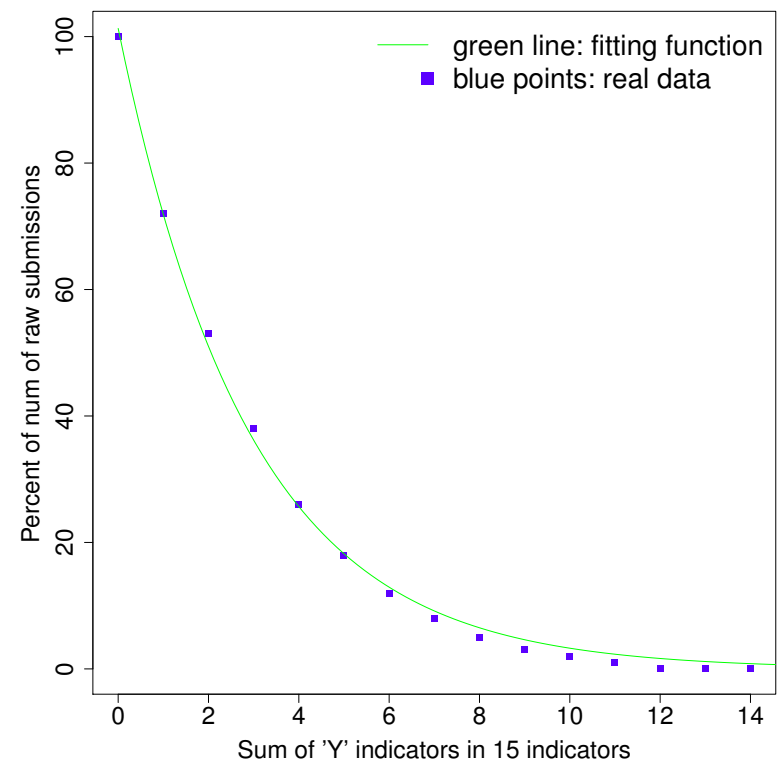

Figure 6: Percent of submissions with at least $K$ indicators, considering indicators 1-15.

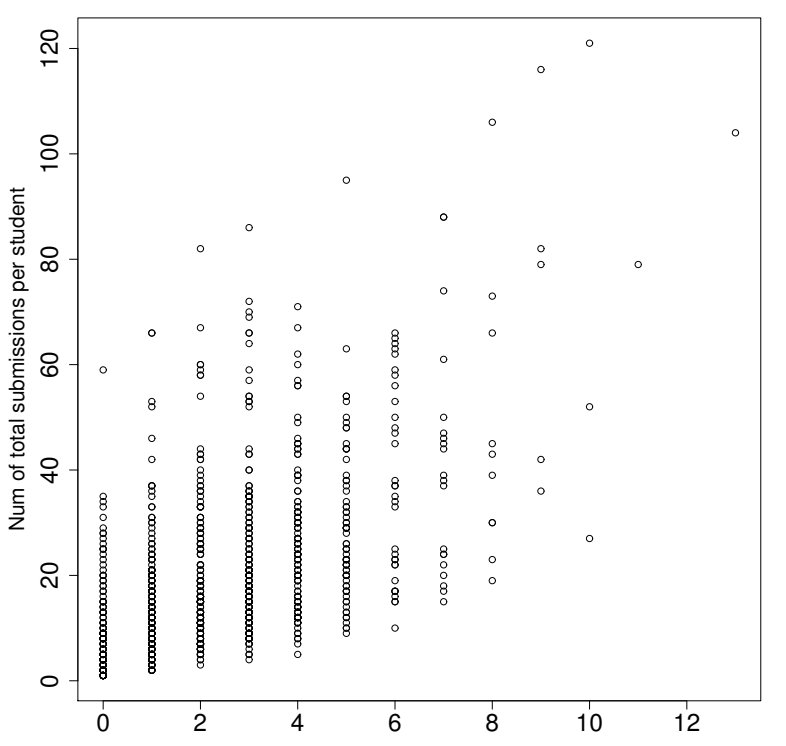

Num of raw submissions with $>=6$ ' $Y$ ' indicators in total submissions per student

Figure 7: Relationship between the total number of submissions, and the number of submissions with at least $K=6$ indicators triggered per student. 


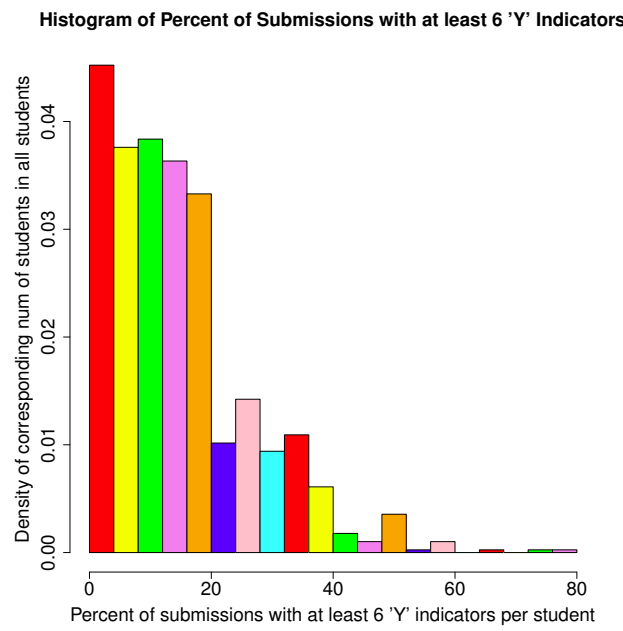

Figure 8: Proportion of submissions triggering $K=$ 6 , plotted against percentage of students.

s.d.), of which $0-6$ submissions meet the $K=6$ criterion. Visually, this region of the scatter plot suggests only a minor relationship between total number of submissions and the subset that triggers the composite measure. This behavior suggests that a triangulated score of at least 6 simultaneous indicators is somewhat conservative, likely having fewer type I errors, but still recognizes some kinds of progress across a wide number of students with differing programming habits.

Unfortunately, increasing $K$ results in an even smaller number of submissions per student achieving the criterion, with most students seeing four or fewer submissions reaching the threshold. While this would decrease (and in the extreme, eliminate) type I errors, it increases type II errors. While a true validation study is still needed, this exploratory analysis suggests $K=6$ as a good candidate value.

\subsection{Individual Student Trajectories}

Aggregating data by student gives a different perspective. Because students made a widely varying number of submissions, some students had only a small percentage (or even none) of their submissions trigger sufficient indicators, while others had a much higher percentage of submissions reach this level-as much as $50 \%$ or more.

Figure 8 shows the distribution of students against the percentage of their submissions that reached at least $K=6$ indicators. To validate the performance of the composite metric, we randomly selected students across this distribution. From Figure 8, we randomly selected five students each with $5 \%, 10 \%$, and $25 \%$ of their submissions reaching at least $K=6$ indicators. Figures 9-11 plot a subset of selected student's submissions against the number of indicators triggered. Each subplot represents the details for all submissions by one individual student from this group. In each subplot, the horizontal axis shows the time of each submission relative to the period between the student's first submission and last submission. While students started and finished at different times, the time period between first and last submission has been normalized to 1.0 in each plot for comparison purposes. The vertical axis shows the number of indicators triggered for the corresponding submission. In
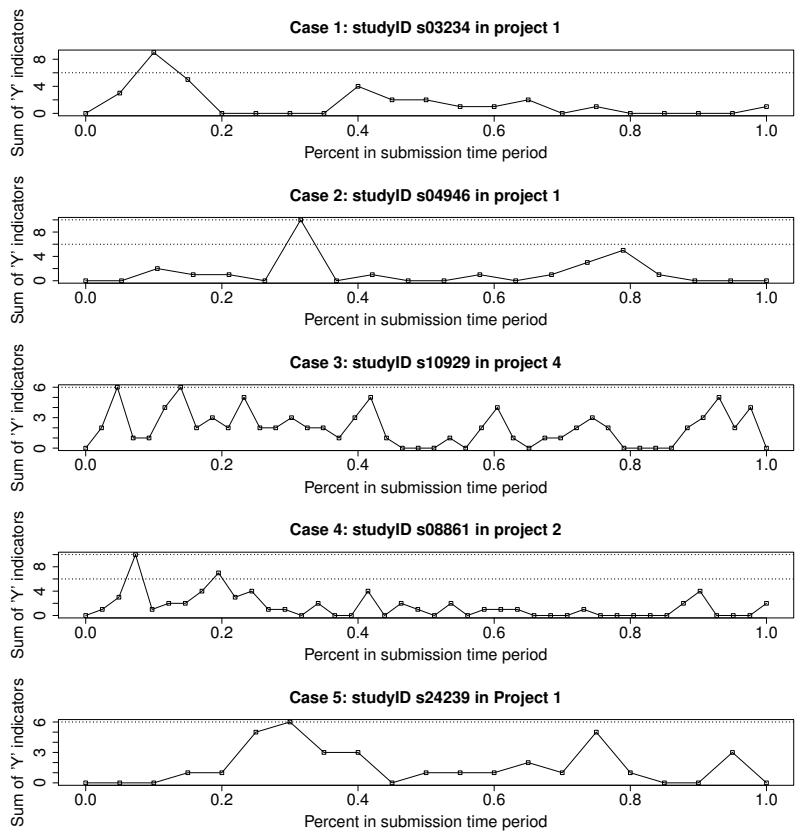

Figure 9: Selected students with $5 \%$ qualifying submissions. Each point represents one submission.

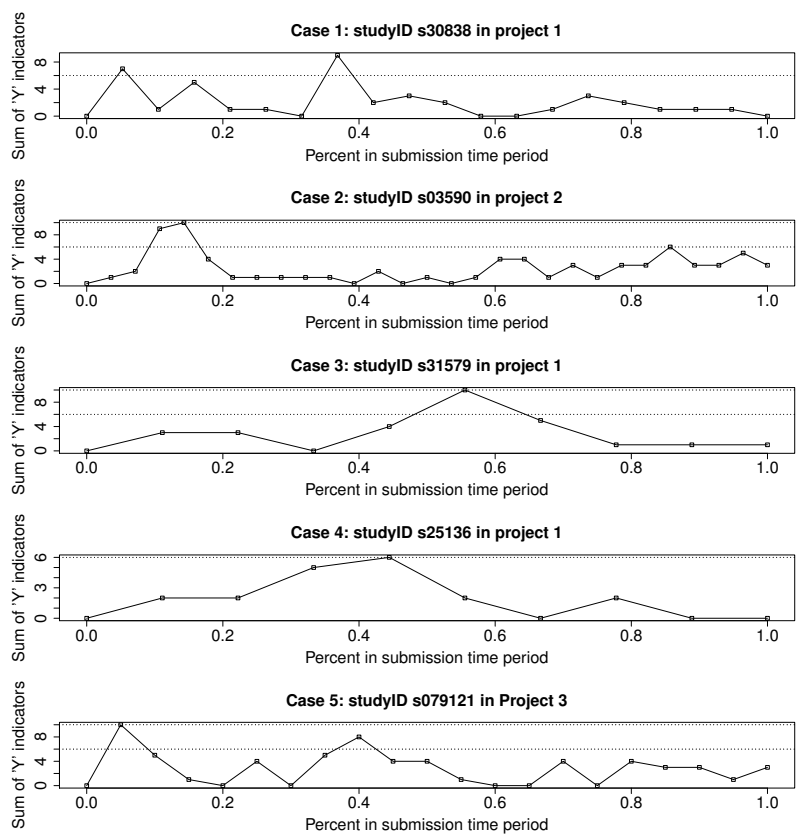

Figure 10: Selected students with $10 \%$ qualifying submissions. Each point represents one submission. 

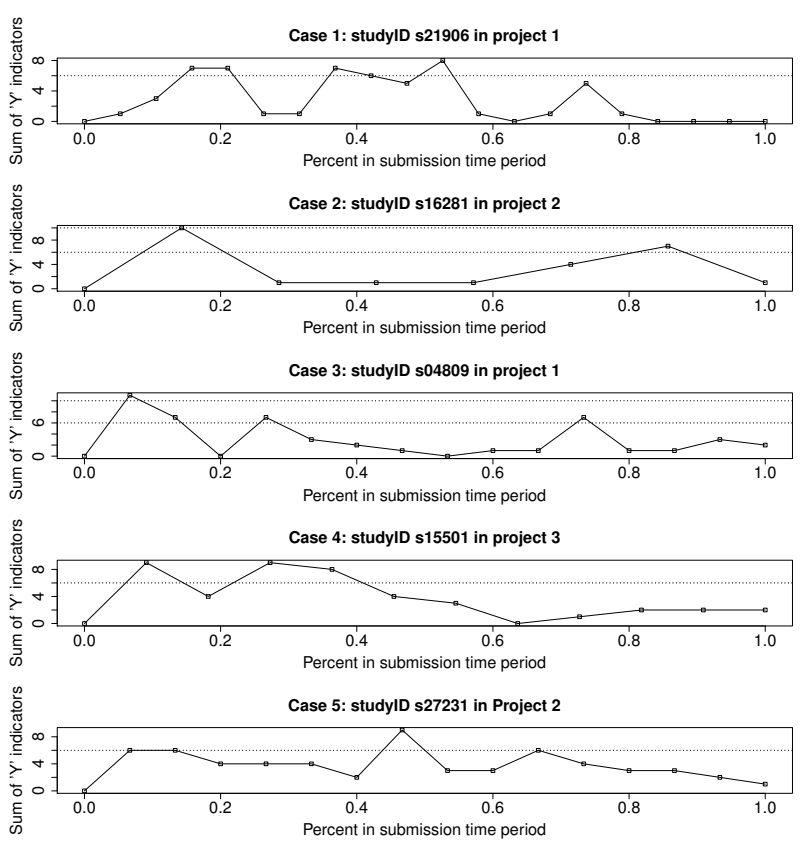

Figure 11: Selected students with 25\% qualifying submissions. Each point represents one submission.

each subplot in Figures 9-11, we also add two horizontal lines with values 6 and 8 as cutting points for the set of triggered indicators, for illustration purposes.

The selected students typically had similar numbers of submissions that reach the $K=6$ threshold. For students who make fewer submissions overall, this represents a higher percentage of their attempts, while for students who make more submissions, a similar number reaching the threshold corresponds to a smaller percentage. In fact, the individual trajectories here correspond directly to Figure 7, where a large number of students with a wide variety of submission counts have relatively little variation in the number of submissions that trigger at least $K=6$ indicators.

To validate the composite measure, we compared the source code by hand for submissions triggering at least 6 indicators against the corresponding previous submissions. This comparison confirmed that students had made substantive changes in every case where the composite measure was triggered, rather than minor tweaks or small adjustments. The composite decision reached by the indicators was accurate with respect to their code. Further, while many submissions under consideration were associated with increased correctness scores, a significant portion were not. The Spearman's rho correlation between when submissions increased their correctness score and when the $K=6$ composite measure was triggered was $\rho=0.27$. This indicates the progress measure is carrying significant information that is not accounted for when considering only program correctness.

\section{CONCLUSIONS}

This preliminary investigation shows that creating indicators to measure student effort and hard work is possible by using historical data for validation. These indicators concentrate on measuring students' progress in their solution development process, based on their assignment submissions.
The indicators in this set are split evenly between those that focus on constructing a solution, which are applicable to any programming assignment, and those that focus on software testing activities. Use of either the more general subset of seven indicators, or the full set of fifteen is viable, and both sets behave in similar ways on authentic student submissions. Together, this feasibility study demonstrates that this approach is practical to apply with existing tools, and resolves the issues of imprecise, noisy measures using triangulation and rolling maxima to increase accuracy while making attempts by students to "game" the system by artificially manipulating measures difficult to achieve.

\subsection{Towards Feedback}

One important area of future development is feedback generation. Feedback plays an important role in the learning process. Appropriate feedback mechanism may encourage the student to practice more. We wish to use this measurement approach as a basis for providing more frequent reinforcing feedback in order to foster a growth mindset.

The indicators developed here provide a basis for generating feedback, regardless of the number that trigger. For individual indicators that trigger, it is possible to generate recognition-oriented feedback that validates a student's efforts. Comments such as "nice job improving your tests" or "paying more attention to comments really helps" can be driven directly from indicators, even if the selected $K$ threshold is not met. Complimentary statements that directly validate effort invested can be driven by reaching the selected $K$ threshold. Task-oriented rewards targeted at increasing intrinsic motivation can also be considered.

Indicators that do not trigger also can be used to generate encouragement-oriented feedback, such as "adding a well-chosen test case is a great way to self-check your work" or "fixing static analysis errors will improve your score right away". While it would be unwise to give feedback about every indicator, or use the same phrasing on every submission, randomly selecting a small number of indicators (especially ones that have changed, or that have been repeatedly missed) for feedback may provide powerful reinforcement.

\subsection{Future Work}

This initial study focused only on the feasibility of using measures to evaluate progress that could be used to drive new feedback strategies. Next steps include a more thorough validation study that uses a larger number of randomly selected student histories to measure the accuracy and error rates of the composite threshold-based measure, and if feasible, the individual indicators. In addition, because the proposed indicators are directly supported by static and dynamic data that are already collected by tools such as Web-CAT, implementation of the indicators themselves is straightforward. A direct implementation path opens up possibilities for designing specific feedback strategies built on these indicators, and then deployment of those new strategies for evaluation with students participating in live courses.

While designing the indicators and evaluating them for feasibility is only the first step toward feedback to encourage a growth mindset, it is a critical element underpinning a successful intervention. One goal of this study is to raise awareness of alternative approaches to encouraging students through psychologically motivated feedback, rather than relying solely on performance-based analysis of work. 


\section{ACKNOWLEDGMENTS}

This work is supported in part by the National Science Foundation under grants DUE-1625425 and DUE-1245589. Any opinions, findings, conclusions, or recommendations expressed in this material are those of the authors and do not necessarily reflect the views of the National Science Foundation.

\section{REFERENCES}

[1] A. Allevato, M. Thornton, S. H. Edwards, and M. Pérez-Quiñones. Mining data from an automated grading and testing system by adding rich reporting capabilities. In Proc. 1st Int'l Conf. Educ. Data Mining, pages 167-176, Montreal, Canada, Jun. 2008.

[2] J. Aronson, C. B. Fried, and C. Good. Reducing the effects of stereotype threat on african american college students by shaping theories of intelligence. $J$. Experimental Social Psychology, pages 113-125, 2002.

[3] L. J. Barker, K. Garvin-Doxas, and M. Jackson. Defensive climate in the computer science classroom. SIGCSE Bull., 34(1):43-47, Feb. 2002.

[4] L. S. Blackwell, K. H. Trzesniewski, and C. S. Dweck. Implicit theories of intelligence predict achievement across an adolescent transition: A longitudinal study and an intervention. Child Development, 78(1):246-263, 2007.

[5] K. Buffardi. Understanding and persuading adherence to test-driven development. In Proc. 9th Ann. Int'l Conf. Int'l Comput. Educ. Research, ICER '12, pages 155-156, New York, NY, USA, 2012. ACM.

[6] K. Buffardi and S. H. Edwards. Impacts of adaptive feedback on teaching test-driven development. In Proc. 44th ACM Tech. Symp. Comput. Sci. Educ., SIGCSE '13, pages 293-298, New York, NY, USA, 2013. ACM.

[7] K. Buffardi and S. H. Edwards. Responses to adaptive feedback for software testing. In Proc. 2014 Conf. Innovation $\&$ Technology in Comput. Sci. Educ., ITiCSE '14, pages 165-170, New York, NY, USA, 2014. ACM.

[8] E. L. Deci, R. Koestner, and R. M. Ryan. A meta-analytic review of experiments examining the effects of extrinsic rewards on intrinsic motivation. Psychological Bull., 125(6):627, 1999.

[9] C. Dweck. Carol dweck revisits the 'growth mindset'. http://www.edweek.org/ew/articles/2015/09/23/ carol-dweck-revisits-the-growth-mindset.html, 2016. Accessed: 2016-08-01.

[10] C. S. Dweck. Motivational Processes Affecting Learning. American Psychologist, 41(10):1040-1048, Oct. 1986.

[11] C. S. Dweck. Self-theories: Their role in motivation, personality and development. Taylor \& Francis, Philadephia, PA, 1999.

[12] C. S. Dweck and E. L. Leggett. A social-cognitive approach to motivation and personality. Psychological Review, pages 256-273, 1988.

[13] S. H. Edwards. Improving student performance by evaluating how well students test their own programs. J. Educ. Resour. Comput., 3(3), Sept. 2003.

[14] S. H. Edwards. Using software testing to move students from trial-and-error to reflection-in-action. In
Proc. 35th SIGCSE Tech. Symp. Comput. Sci. Educ., SIGCSE '04, pages 26-30, New York, NY, USA, 2004. ACM.

[15] A. Fisher and J. Margolis. Unlocking the clubhouse: The carnegie mellon experience. SIGCSE Bull., 34(2):79-83, June 2002.

[16] A. E. Flanigan, M. S. Peteranetz, D. F. Shell, and L.-K. Soh. Exploring changes in computer science students' implicit theories of intelligence across the semester. In Proc. 11th Ann. Int'l Conf. Int'l Comput. Educ. Research, pages 161-168, New York, NY, USA, 2015. ACM.

[17] H. Grant and C. S. Dweck. Clarifying achievement goals and their impact. J. Personality 85 Social Psychology, pages 541-553, 2003.

[18] B. Hanks, L. Murphy, B. Simon, R. McCauley, and C. Zander. Cs1 students speak: Advice for students by students. SIGCSE Bull., 41(1):19-23, Mar. 2009.

[19] G. D. Heyman, B. Martyna, and S. Bhatia. Gender and achievement-related beliefs among engineering students. J. Women and Minorities in Sci. and Eng., $8(1): 41-52,2002$.

[20] T. E. Higgins and W. S. Rholes. "saying is believing": Effects of message modification on memory and liking for the person described. J. Experimental Social Psychology, 1978, 14(4):363-78, 1978.

[21] P. Ihantola, T. Ahoniemi, V. Karavirta, and O. Seppälä. Review of recent systems for automatic assessment of programming assignments. In Proc. 10th Koli Calling Int'l Conf. Comput. Educ. Research, pages 86-93, New York, NY, USA, 2010. ACM.

[22] C. Lewis. Attitudes and beliefs about computer science among students and faculty. SIGCSE Bull., 39(2):37-41, June 2007.

[23] C. M. Lewis, K. Yasuhara, and R. E. Anderson. Deciding to major in computer science: A grounded theory of students' self-assessment of ability. In Proc. '7th Int'l Workshop on Comput. Educ. Research, ICER '11, pages 3-10, New York, NY, USA, 2011. ACM.

[24] L. Murphy and L. Thomas. Dangers of a fixed mindset: Implications of self-theories research for computer science education. In Proc. 13th Ann. Conf. Innovation and Tech. in Comput. Sci. Educ., pages 271-275, New York, NY, USA, 2008. ACM.

[25] E. O'Rourke, Y. Chen, K. Haimovitz, C. S. Dweck, and Z. Popović. Demographic differences in a growth mindset incentive structure for educational games. In Proc. Second (2015) ACM Conf.Learning@ Scale, pages 331-334, New York, NY, USA, 2015. ACM.

[26] E. O'Rourke, K. Haimovitz, C. Ballweber, C. Dweck, and Z. Popović. Brain points: A growth mindset incentive structure boosts persistence in an educational game. In Proc. 32nd Ann. ACM Conf. Human Factors in Comput. Sys., pages 3339-3348, New York, NY, USA, 2014. ACM.

[27] B. Simon, B. Hanks, L. Murphy, S. Fitzgerald, R. McCauley, L. Thomas, and C. Zander. Saying isn't necessarily believing: Influencing self-theories in computing. In Proc. Fourth Int'l Workshop on Comput. Educ. Research, pages 173-184, New York, NY, USA, 2008. ACM. 